\title{
BOOKED ADMISSIONS: A NEW REFERRAL SYSTEM FOR GENERAL DENTAL PRACTITIONERS IN MORECAMBE BAY
}

\author{
Peter Dyer, Consultant Oral and Maxillofacial Surgeon \\ Morecambe Bay Hospitals NHS Trust
}

\section{INTRODUCTION}

A key tenet of a modern patient-centred health service is that patients can book their hospital appointments for a time that is convenient to them, and since November 1998, rollout of the National Booked Admissions Programme has encouraged the development of many schemes to facilitate this. The first wave of the programme focused on day surgery and this was followed in the second wave by inpatients, diagnostic services, physiotherapy and endoscopy.

'Delivering 21 st Century IT Support for the NHS'(1) sets out a programme for providing a common IT link between the many different facets of the NHS. The future role of dentistry within the NHS is discussed in 'NHS Dentistry: Options for Change' ${ }^{(2)}$ and again the importance of IT is emphasised by the Department of Health paper published in October 2002, 'An information Technology Strategy for NHS Dentistry in the 21st Century ${ }^{(3)}$.

This article reports on the experience of a booked admissions scheme (now known as eBooking) in Morecambe Bay involving the Department of Oral and Maxillofacial Surgery, serving three hospitals in the acute trust and three general dental practices. This scheme, which is the first to involve dentists and their patients, has been established for more than a year and illustrates the importance of IT in dentistry.

\section{BACKGROUND}

Morecambe Bay Hospitals NHS Trust was established in 1998 with the amalgamation of the trusts at the Royal Lancaster Infirmary, Furness General Hospital in Barrow and Westmorland General Hospital in Kendal. The trust covers an area of 3000 square miles, serving a mixed urban and rural population of over 300,000 . There are 53 dental practices and 112 dentists in the area referring patients to the Department of Oral and Maxillofacial Surgery with problems ranging from difficult dental extractions to suspected oral cancer.

Traditionally, referrals were sent by letter to the nearest hospital within the trust or by telephone in the case of an emergency. Approximately $65 \%$ of referrals were from dental practices and the remainder from general medical practices or were tertiary referrals from within the hospital. Having received and read the letter, the consultant graded them as routine, soon or urgent and reception staff allocated an outpatient clinic slot before contacting the patient. Previous studies have shown that given appropriate guidelines, general dental practitioners were able to refer patients directly to hospital for oral surgery procedures, bypassing the outpatient appointment and thus reducing the number of visits to the hospital ${ }^{(4.5)}$ although uptake of the new referral system in one of the studies was low ${ }^{(5)}$, reflecting the reluctance to change wellestablished patterns. Interestingly, a further study ${ }^{(6)}$ demonstrated that dentists were happy to incorporate clinical algorithms into their traditional referral practice but were reluctant to use computer-based packages.

\section{METHODOLOGY}

Funding for this project was provided as part of the third wave of the National Booked Admissions Programme and in the first instance it was decided to link with three dental practices, which were geographically distributed around Morecambe Bay to represent Lancaster, Barrow and Kendal. The Local Dental Committee was asked to inform dentists about the scheme and to choose the participating practices, with the only other criteria being that the practices should be mainly NHS.

Process mapping of the dental practices revealed that each had an established referral pathway and for one this involved over 30 different stages. For the first year of the project referrals from the practices for three selected problems (wisdom teeth, periapical pathology and difficult extractions) were made in paper form on a proforma. By following the clinical algorithm on the proforma the referring dentists could decide whether the patient needed an outpatient consultation or could be given an appointment directly for treatment. The proforma established the use of algorithms and also provided a means for tracking the referrals.

The scheme went live in January 2002. The three dental practices were linked to the Department of Oral and Maxillofacial Surgery by the NHSNet; two practices using ISDN lines and one using Kilostream. A firewall was established between the practices and the hospitals for security purposes. Each practice was provided with the necessary IT equipment and training sessions were held for all the participating dentists.

Referral protocols were written for the following conditions:

- difficult extractions

- third molar teeth (wisdom teeth)

- periapical pathology (teeth with an abscess)

- facial swellings

- salivary gland problems

- oral cancer

- facial pain

Each protocol was written to incorporate the latest developments in evidence-based dentistry and the participating dentists were involved in their development. For example, if referrals for third molar surgery did not follow NICE guidelines 
the booking was prevented. From their surgery, with the patient still present, the dentist, by following the protocol, could make an appointment at the nearest hospital at a time convenient for the patient. The dentist could choose an outpatient clinic appointment or a treatment slot if appropriate. If the latter, the practitioner could also print off pre-operative instructions for the patient relating to the planned procedure. Dedicated clinic slots were established for booked admissions patients and these were available for the dentist to fill accordingly.

Urgent referrals could be made but the dentists were advised that they could still telephone the department if necessary.

The NHSNet already linked most general medical practices to the hospitals and so they had the facility to refer patients to the department using the protocols.

\section{RESULTS}

The referral pattern to the hospital is shown in the figure below. Forty-six electronic referrals were made between January 2002 and March 2003. Forty-four referrals were made by the participating dental practices and a further two were from a general medical practice. Two referrals for suspected oral cancer ensured that the patients were seen within the two-week wait period.

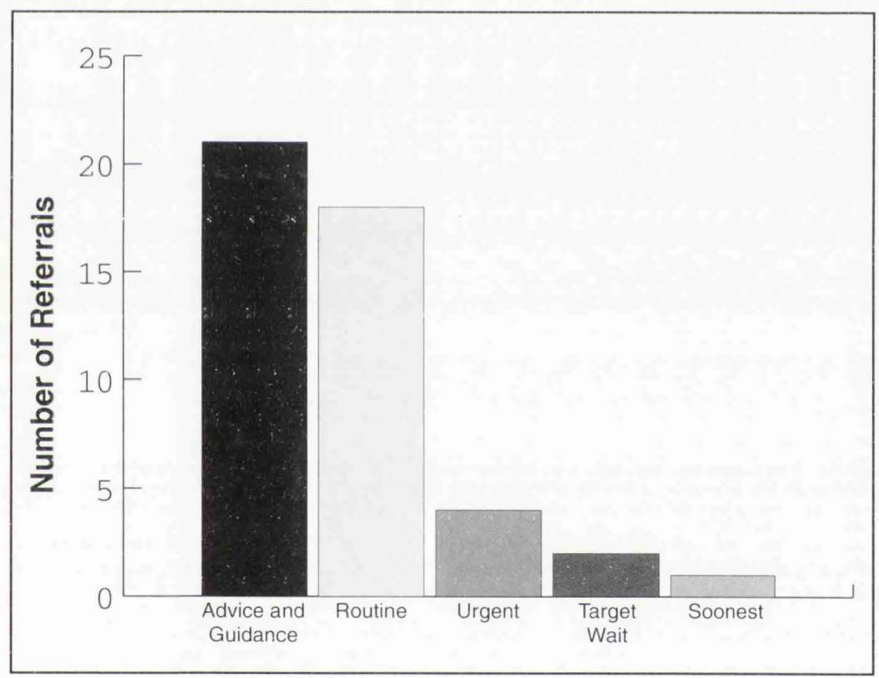

Electronic referrals to the Department of Oral and Maxillofacial Surgery priority type January 2002 - March 2003

\section{DISCUSSION}

Dentistry is an integral part of the NHS and there is a clear commitment to incorporate IT into the routine working practise of dentists. This scheme has demonstrated that IT can be used to link the primary and secondary care elements of dentistry to facilitate the patient journey and improve their experience.

Several challenges have arisen from this scheme and are being addressed. The results showed that a large number of referrals were ending in 'advice and guidance'. As a result a new protocol was established for advice and guidance only. One protocol (periapical pathology) was rewritten because the initial version was too difficult to follow.

A number of practical issues have been identified which must be considered if dentists are going to use IT more fully in the future. Dentists tend to work exclusively in their surgeries and the geographical layout of computers in a practice must be considered. Ideally, each dentist should have a computer in his/her surgery, with another in the reception area. One practice was already using computers to link with the Dental Practice Board in Eastbourne. For security reasons, the booked admissions need to be made on equipment provided by the trust. Difficulties may arise in terms of space in the dental surgery if two computers are next to each other and with regard to the compatibility of the existing software and the new equipment.

Radiographs remain an important issue. In both the preliminary paper exercise and the final IT version, radiographs were brought to the hospital by the patient or sent by the dentist. It is hoped that eventually the radiographic images will be sent via the net together with the referral.

The most common cause of concern for the dentists was the length of time spent following the protocol to make a referral. One dentist made the point that it was much quicker to dictate a referral letter than to use valuable clinical time to sit at the computer screen. This dentist used to wait until the practice was closed before referring the patient. The disadvantage of this was that the dentist could not discuss appointment times with the patient. A different solution in one of the other practices was to send the patient's details through to reception and ask the receptionist to negotiate the appointment time.

Finally, one of the practitioners admitted to a 'phobia of computers' and this is clearly a very real problem when introducing new methods.

\section{THE FUTURE}

There are plans for two main developments in Morecambe Bay regarding dentistry and IT. The first, which is to increase the number of practices involved, has already taken a step forward as a result of funding through the Modernising Dentistry programme. Two more practices will go live in the next few months.

Secondly it is hoped that the Integrated Care Pathways which have been in use by the department for almost three years (in minor oral surgery, paediatric oral surgery and facial skin lesions) could be included as part of booked admissions. The current practice is for the pathways to be completed in the hospital clinic and kept with the patient's notes. As electronic patient records are developed it is envisaged that the care pathways could be started in the dental surgery and sent to the hospital with the referral and thus be available on a computer screen in the outpatient clinic, creating a truly seamless journey for the patient from primary to secondary care.

Acknowledgements: I would like to thank all the members of the Booked Admissions team in the Morecambe Bay Hospitals NHS Trust for their help and support in establishing this scheme with the local general dental practitioners.

\section{REFERENCES}

1 Delivering 21 st Century IT Support for the NHS. Department of Health. London. April 2002

2 NHS Dentistry: Options for Change. Department of Health. London. August 2002

3 An Information Technology Strategy for NHS Dentistry in the 21 st Century. Department of Health. London. October 2002 4 Joshi A, Doyle L, Worthington HV, Rood JP. Direct access day case oral surgery. Br Dent J 2000;88:452-456

5 Renton T, McGurk M. Direct referral day case oral surgery for dental practitioners: a pilot investigation. $\mathrm{Br}$ Dent $\mathrm{J}$ 1999;186:334-337

6 Goodey RD, Brickley MR, Hill CM, Shepherd JP. A controlled trial of three referral methods for patients with third molars. Br Dent J 2000;189:556-560 